\title{
Cognitive-Behavior Therapy (CBT) for Panic Disorder: Relationship of Anxiety and Depression Comorbidity with Treatment Outcome
}

\author{
Laura B. Allen • Kamila S. White • David H. Barlow • \\ M. Katherine Shear • Jack M. Gorman • \\ Scott W. Woods
}

Published online: 24 July 2009

(C) The Author(s) 2009. This article is published with open access at Springerlink.com

\begin{abstract}
Research evaluating the relationship of comorbidity to treatment outcome for panic disorder has produced mixed results. The current study examined the relationship of comorbid depression and anxiety to treatment outcome in a large-scale, multi-site clinical trial for cognitive-behavior therapy (CBT) for panic disorder. Comorbidity was associated with more severe panic disorder symptoms, although comorbid diagnoses were not associated with treatment response. Comorbid generalized anxiety disorder (GAD) and major depressive
\end{abstract}

\section{B. Allen $(\bowtie)$}

Department of Pediatrics,

David Geffen School of Medicine at UCLA,

10833 Le Conte Avenue, 22-464 MDCC,

Los Angeles, CA 90095, USA

e-mail: LBAllen@mednet.ucla.edu

\section{K. S. White}

Department of Psychology, University of Missouri-St. Louis,

St. Louis, MO, USA

D. H. Barlow

Department of Psychology, Boston University,

Boston, MA, USA

\section{K. Shear}

Department of Psychiatry,

Columbia University School of Social Work,

New York, NY, USA

J. M. Gorman

Comprehensive NeuroScience, Inc.,

White Plains, NY, USA

S. W. Woods

Department of Psychiatry, Yale University,

New Haven, CT, USA disorder (MDD) were not associated with differential improvement on a measure of panic disorder severity, although only rates of comorbid GAD were significantly lower at posttreatment. Treatment responders showed greater reductions on measures of anxiety and depressive symptoms. These data suggest that comorbid anxiety and depression are not an impediment to treatment response, and successful treatment of panic disorder is associated with reductions of comorbid anxiety and depressive symptoms. Implications for treatment specificity and conceptual understandings of comorbidity are discussed.

Keywords Panic disorder Comorbidity .

Treatment outcome $\cdot$ Anxiety $\cdot$ Depression

Psychiatric comorbidity is typically defined as the cooccurrence of two or more psychiatric disorders within the same individual (Maser and Cloninger 1990). Comorbidity research is particularly important because the majority of individuals presenting for treatment for an Axis I anxiety or mood disorder have at least one additional anxiety or mood disorder diagnosis (e.g., Brown et al. 1995; Brown and Barlow 1992, 1995; de Ruiter et al. 1989; Sanderson et al. 1990). For individuals seeking treatment for panic disorder, comorbidity rates for Axis I disorders generally range from $51 \%$ to $69 \%$ with some of the larger studies suggesting that estimates are approximately $60 \%$, with generalized anxiety disorder (GAD), social phobia, and depression being the most frequently assigned disorders (Brown and Barlow 1992; Brown et al. 1995, 2001a; Sanderson et al. 1990; Tsao et al. 1998, 2002).

Studies examining treatment outcome for panic disorder with anxiety and depression comorbidity have produced mixed results. While a number of studies have suggested 
that the presence of comorbid diagnoses, particularly depression, is associated with less improvement on some measures of panic symptoms (i.e., achieving substantial improvement in panic symptoms or attaining "panic-free" status) (Brown et al. 1995; Steketee et al. 2001; Tsao et al. 1998), these findings have not been consistent. For example, Tsao et al. (2002) found no differences between anxious and depressive comorbid and non-comorbid groups at posttreatment on panic severity, agoraphobia severity, and endstate functioning, and similar findings have been evident when examining comorbid depression, specifically (McLean et al. 1998). In fact, some comorbidity, such as social phobia, may be associated with better treatment outcome for panic disorder (Brown et al. 1995). The mixed outcomes from these studies suggest that no stable relationship between certain types of comorbidity and panic disorder treatment has yet emerged. This may be due in part to variability in assessment measures, as well as the relatively small sample sizes in previous studies (e.g., Tsao et al. 1998, 2002).

A more consistent and encouraging finding is that, overall, comorbid diagnoses show significant reductions over the course of treatment, even though these concerns are not the focus of the treatment provided. With the exception of one study (McLean et al. 1998) that found depressive symptoms changed only at a rate similar to changes expected from the passage of time, results from Brown et al. (1995) and two other studies (Tsao et al. 1998, 2002) report significant reductions in the rates of comorbid GAD, social phobia, and depression from preto posttreatment.

The current study aims to replicate and extend the research on comorbidity in panic disorder by examining the relationship of comorbid anxiety and unipolar mood disorders to treatment outcome. The strengths of this study include (a) a large sample size generated from four study sites, (b) both clinician-rated and self-report measures focusing on panic and associated symptoms, (c) carefully monitored adherence to a manualized CBT protocol for panic disorder, and (d) diagnostic assessments conducted by independent evaluators (IEs). Although the panic disorder and comorbidity literature has not produced consistent findings upon which to base hypotheses, given the data from relatively large samples in previous studies, we hypothesize that rates of comorbidity will approximate those previously reported in studies where panic disorder is the principal diagnosis, with GAD, social phobia, and major depressive disorder (MDD) being the most common comorbid diagnoses. Furthermore, we hypothesize that comorbidity will not be associated with a decreased likelihood of achieving treatment response, and overall rates of comorbid diagnoses will decrease following treatment.

\section{Method}

Participants

Participants were 454 patients who were part of a multi-site, large-scale clinical trial examining long-term treatment strategies for panic disorder (see Aaronson et al. 2008 for full description of study procedures). Of this sample, 379 were enrolled in the study at four different sites: 93 at the Center for Anxiety and Related Disorders at Boston University, 92 at the Hillside Hospital of the North Shore/ Long Island Jewish Health Care System, 103 at the Western Psychiatric Institute and Clinic, and 91 at Yale University. Subsequently, 256 patients completed the acute phase of the study and received a posttreatment diagnostic interview and assessment; 123 patients either dropped out or were removed from the study.

Inclusion criteria were: (a) a principal DSM-IV diagnosis of panic disorder with or without agoraphobia; (b) age 18 years or older; (c) no substance abuse or dependence within the last 6 months; (d) absence of active suicide potential within the last 6 months; (e) absence of any history of psychosis, bipolar I disorder, bipolar II disorder, or cyclothymia; (f) no current application pending or existing for a medical disability claim; (g) no significant cognitive impairment; (h) free from current uncontrolled general medical illness requiring intervention; (i) absence of concurrent psychotherapeutic treatment directed at anxiety and panic disorders and; (j) no concurrent psychopharmacological treatment that may have anti-panic effects (participants were required to taper medication by session 9). Informed consent was obtained from all participants prior to study enrollment. This study was approved by the institutional review boards at all four study sites.

Demographic information for treatment completers is available in Table 1. There were no gender or ethnicity differences between individuals who did and did not complete treatment. However, treatment completers were significantly older $(M=38.4, S D=11.7)$ than noncompleters $(M=34.6, S D=11.6), t(379)=-3.00, p=0.003$. Treatment completers also attended significantly more years of school $(M=15.3, S D=3.0)$ than noncompleters $(M=14.3, S D=2.7)$, $t(372)=-2.94, p=0.004$.

\section{Measures}

ADIS-Lite The Anxiety Disorders Interview Schedule-Lite (ADIS-Lite) is an adaptation of the ADIS for DSM-IVLifetime version (ADIS-IV-L; Di Nardo et al. 1994) and differs only with respect to assessing for the presence of symptoms (yes/no) rather than a dimensional rating for all symptoms $(0-8$ scale). This semi-structured clinical interview focuses on the anxiety, mood, substance use, and somatoform disorders and the ADIS-IV-L has demonstrated 
Table 1 Demographic information for treatment-completing sample $(N=256)$

\begin{tabular}{lc}
\hline Measure & Number (\%) \\
\hline Age (mean) & 38.37 \\
Women & $165(64.5)$ \\
Ethnicity & \\
Caucasian & $224(87.5)$ \\
African-American & $14(5.5)$ \\
Asian or Pacific Islander & $14(5.5)$ \\
American Indian or Alaskan Native & $3(1.2)$ \\
Other & $1(0.4)$ \\
Marital status & \\
Never married & $93(36.3)$ \\
Married & $134(52.4)$ \\
Separated or divorced & $28(10.9)$ \\
Number of children (mean) & 1.14 \\
Years attended school (mean) & 15.25 \\
\hline
\end{tabular}

excellent to acceptable interrater reliability for the anxiety and mood disorders (Brown et al. 2001b).

IEs across the four sites underwent a rigorous certification procedure to ensure standardization of administration and diagnostic reliability, and were blind to participants' progress. Ten percent of all assessments were randomly selected for diagnostic co-rating. Intraclass correlation coefficients were calculated to assess interrater reliability, which was acceptable to excellent across the diagnoses (panic disorder $\rho_{I}=0.885$, agoraphobia $\rho_{I}=0.658$, MDD $\rho_{I}=0.99$, GAD $\rho_{I}=0.999$, obsessive compulsive disorder $\rho_{I}=0.747$, social phobia $\rho_{I}=0.909$, specific phobia $\rho_{I}=0.795$, dysthymia $\rho_{I}=0.489$, and posttraumatic stress disorder $\rho_{I}=0.486$ ).

Agoraphobia Scale IEs rated panic-related fear and avoidance of particular situations, ranging from 0 (no fear/never avoids) to 4 (extreme fear/always avoids). This measure has demonstrated excellent internal consistency ( $\alpha=0.87$ in our sample) and is associated with excellent interrater reliability $(r=0.86$, Brown et al. 2001b).

Panic Disorder Severity Scale-Independent Evaluator Version (PDSS-IE; Shear et al. 1992, 1997) The PDSS-IE is a 7-item measure for assessing the frequency, distress, and avoidance caused by panic attacks. Each item is rated on a 0 (none/mild) to 4 (extreme/severe) scale. Interrater reliability $\left(N=434, \rho_{I}=0.993\right)$ and internal consistency $(\alpha=0.81)$ were both excellent in the current study.

Structured Interview Guide for the Hamilton Anxiety Rating Scale and Structured Interview Guide for the Hamilton
Depression Rating Scale (SIGH-A and SIGH-D) (Shear et al. 2001; Williams 1988) The SIGH-A and SIGH-D were developed to create a structured format for administering the Hamilton Anxiety Rating Scale (Hamilton 1959) and Hamilton Rating Scale for Depression (Hamilton 1960), respectively. The SIGH-A and SIGH-D have each demonstrated good inter-rater and test-retest reliability. In the current study, the SIGH-A demonstrated excellent interrater reliability and internal consistency $\left(N=193, \rho_{I}=0.990\right.$, $\alpha=0.86)$, as did the SIGH-D $\left(N=181, \rho_{I}=0.997, \alpha=0.84\right)$.

Anxiety Sensitivity Index (ASI; Peterson and Reiss 1987) The ASI is a 16-item self-report scale used to evaluate anxiety focused on panic-related bodily sensations. It has been used extensively in research on panic disorder and demonstrated excellent internal consistency $(\alpha=0.88)$ in our sample.

Work and Social Adjustment Scale (WSAS; a modification of a scale introduced by Hafner and Marks 1976) The WSAS is a five-item measure that asks participants to rate the degree of interference caused by their symptoms in five life domains on a 0 (no interference) to 8 (very severe interference) scale. This measure has shown adequate testretest reliability $(r=0.73)$ (Mundt et al. 2002) and good internal consistency $(\alpha=0.80)$ in our sample.

\section{Measures of Treatment Response}

Criteria for treatment responder status were set as part of the larger clinical trial (Aaronson et al. 2008). Criteria for treatment response were: (a) receive a score of " 2 " (much improved) or "1" (very much improved) on the Clinical Global Impressions scale (CGI; Guy 1976), as determined by the IE at the posttreatment assessment and (b) evidence at least a $40 \%$ reduction in the PDSS-IE score from pretreatment. We also included an additional, more stringent measure of functioning: panic-free status (i.e., having no full or limited symptom panic attacks in the past month), as used in the Brown et al. (1995) study. The first question of the PDSS-IE assessed for the presence of any panic attacks in the past month.

\section{Procedure}

The acute study phase consisted of eleven individual sessions of CBT for panic disorder that was delivered via a manualized protocol (a modified version of the standard Panic Control Treatment; Barlow and Craske 2007). Following treatment, participants were then randomized to additional arms of the study (reports are in preparation). Responders were randomized to receive either (a) no additional treatment 
or (b) nine monthly maintenance CBT sessions; all were followed for a total of 21 months posttreatment. Nonresponders were randomized to receive either (a) an additional course of CBT for panic disorder (3 months) or (b) a trial of paroxetine (lasting from 3-12 months) and participants in each nonresponder group were followed for 12 months total. Due to the addition of different treatments following post-acute CBT sessions, the current paper focuses only on the acute phase of treatment where all participants received CBT.

\section{Data Analyses}

All patients who completed acute treatment were included in analyses $(N=256)$. Intent-to-treat analyses were not conducted, given that the measures used were administered only at pre- and posttreatment. For all data analyses, a modified Bonferroni adjustment was applied to control for Type I error based on the number of pretreatment comparisons. Twenty-four pretreatment comparisons were conducted ( $24 t$-tests and 4 correlations), such that analyses resulting in a significance value of 0.0021 or less were considered significant $(0.05 / 24=0.0021)$. This criterion was then applied to all analyses to determine significance.

\section{Results}

Analyses were first conducted to examine pretreatment differences in rates of comorbidity between treatment completers and noncompleters (see Table 2). No significant differences were found. A detailed paper of study attrition is currently under review (White et al. 2009, Attrition in a multi-center clinical trial for panic disorder. Unpublished manuscript). Of treatment completers, those with comorbidity $(M=40.3, S D=11.9)$ were significantly older $(M=36.8$, $S D=11.2)$ than those without comorbidity $t(254)=-2.44$, $p<0.5$. The same was true for those with $(M=40.3, S D=$ $11.9)$ and without $(M=40.3, S D=11.9)$ comorbid $G A D$, and with $(M=40.3, S D=11.9)$ and without $(M=40.3, S D=11.9)$ comorbid specific phobia. Age was not significantly associated with any other comorbid diagnosis or measure of panic disorder severity.

Rates of Comorbidity at Pretreatment

Table 2 describes the pattern of comorbidity in the treatment completing sample $(N=256)$. Of note, the rate of comorbid MDD appears slightly low, given rates reported in other large-scale studies, e.g., Brown et al. (2001a) reported that of patients with principal panic disorder, $31 \%$ also had a comorbid mood disorder. However, the rate of MDD reported in the current study is similar to those reported in other studies focusing solely on the principal diagnosis of panic disorder: 12.7\% (Brown et al. 1995) and $21.2 \%$ (Tsao et al. 1998).

Severity of Panic Symptoms at Pretreatment

The presence of at least one comorbid diagnosis was associated with greater severity on all four measures of panic disorder and agoraphobia symptoms, as shown in Table 3. Regarding the most common comorbid diagnoses, individuals with comorbid GAD at pretreatment reported higher PDSS-IE scores $t(254)=-3.44, p<0.001, d=0.48$ and ASI scores $t(254)=-3.32, p<0.001, d=0.47$. Comorbid social
Table 2 Rates of pretreatment comorbidity for treatment completers and noncompleters

$G A D$ generalized anxiety disorder; $M D D$ major depressive disorder; $O C D$ obsessivecompulsive disorder; PTSD posttraumatic stress disorder.

\begin{tabular}{lcc}
\hline Pretreatment variable & Completers $(n=256)$ & Noncompleters $(n=123)$ \\
\cline { 2 - 3 } & Number $(\%)$ of patients & Number (\%) of patients \\
\hline Any comorbidity & $117(45.7 \%)$ & $68(55.3 \%)$ \\
Any Anxiety & $99(38.7 \%)$ & $60(48.9 \%)$ \\
Any depression & $48(18.8 \%)$ & $24(19.5 \%)$ \\
GAD & $65(25.4 \%)$ & $39(31.7 \%)$ \\
MDD & $42(16.4 \%)$ & $20(16.3 \%)$ \\
Specific phobia & $30(11.7 \%)$ & $22(17.9 \%)$ \\
Social phobia & $28(10.9 \%)$ & $20(16.3 \%)$ \\
OCD & $15(5.9 \%)$ & $5(4.1 \%)$ \\
PTSD & $10(3.9 \%)$ & $11(8.9 \%)$ \\
Hypochondriasis & $9(3.5 \%)$ & $6(4.9 \%)$ \\
Dysthymia & $8(3.1 \%)$ & $5(4.1 \%)$ \\
1 comorbid diagnosis & $58(22.7 \%)$ & $25(20.3 \%)$ \\
2 comorbid diagnoses & $38(14.8 \%)$ & $31(25.2 \%)$ \\
3 comorbid diagnoses & $14(5.5 \%)$ & $8(6.5 \%)$ \\
4 or more comorbid diagnoses & $7(2.7 \%)$ & $4(3.3 \%)$ \\
\hline
\end{tabular}


Table 3 Means (standard deviations) of pretreatment panic and agoraphobia measures for COM and NOCOM groups

\begin{tabular}{lccc}
\hline Measure & COM $(n=117)$ & $\operatorname{NOCOM}(n=139)$ & ES \\
\hline PDSS-IE & $15.45(4.55)$ & $12.50(3.94)^{\mathrm{a}}$ & 0.66 \\
Agoraphobia Scale & $20.99(14.42)$ & $14.78(11.30)^{\mathrm{a}}$ & 0.47 \\
ASI & $38.57(10.31)$ & $30.80(12.21)^{\mathrm{a}}$ & 0.65 \\
WSAS & $3.33(1.82)$ & $2.55(1.59)^{\mathrm{a}}$ & 0.45 \\
\hline
\end{tabular}

$\mathrm{COM}=$ individuals with at least one comorbid diagnosis at pretreatment, $\mathrm{NOCOM}=$ individuals with no comorbidity

$E S$ effect size (Cohen's $d$ ); PDSS-IE panic disorder severity scaleindependent evaluator version; $A S I$ anxiety sensitivity index; WSAS work and social adjustment scale

a indicates difference from COM at $p<0.01$

phobia at pretreatment was associated with higher ASI scores $t(254)=-3.14, p<0.002, d=0.62$. Comorbid MDD was also associated with significantly greater panic disorder severity across all measures: PDSS-IE, $t(254)=-6.67, p<0.001$, $d=1.04$, Agoraphobia Scale, $t(52.31$, adjusted for unequal variances $)=-3.98, p<0.001, d=0.74$, ASI, $t(254)=-3.65$, $p<0.001, d=0.60$, and WSAS, $t(254)=-6.14, p<0.001$, $d=0.97$. Number of comorbid diagnoses at pretreatment was significantly correlated to PDSS-IE scores $(r=0.37$, $p<0.001)$, Agoraphobia Scale scores $(r=0.24, p<0.001)$, ASI scores $(r=0.31, p<0.001)$, and WSAS scores $(r=0.26$, $p<0.001)$. No significant differences on pretreatment measures of panic disorder were found for individuals with comorbid specific phobia at pretreatment.

\section{Pretreatment Comorbidity and Treatment Response}

The most common comorbid diagnoses at pretreatment were compared to measures of treatment outcome (i.e., treatment responder/nonresponder, panic-free/not panic-free). GAD, MDD, social phobia, or specific phobia at pretreatment was not associated with differential rates of treatment response or individuals achieving panic-free status.

Comorbid Anxiety and Depression and Changes in Panic Symptoms Across Treatment

Two (comorbidity: present v. absent) $\times 2$ (time: pre v. post) repeated measures ANOVAs were conducted across PDSS-IE scores for each of the most common comorbid anxiety and depressive disorders (GAD and MDD). For participants with and without comorbid GAD, a significant time effect was found $F(1,254)=393.74, p<0.001, \eta_{\mathrm{p}}^{2}=0.61$. However, there were no significant group or interaction effects on this variable $\left(\eta_{\mathrm{p}}^{2}=0.04\right.$ and 0.01 , respectively). For those with and without comorbid MDD, analysis of the PDSS-IE revealed a significant effect of time $\quad F(1,254)=333.48, p<$ $0.001, \eta_{\mathrm{p}}^{2}=0.57$ and group $F(1,254)=34.87, p<0.001$, $\eta_{\mathrm{p}}^{2}=0.12$; however, no significant group $\mathrm{x}$ time interaction effect emerged $\left(\eta_{\mathrm{p}}^{2}=0.03\right)$.

The large sample size of this study allowed additional analyses to be conducted by separating participants into four groups: no comorbid diagnosis of any kind $(n=139)$, only anxiety comorbidity (including individuals with any comorbid anxiety disorder such as GAD, social phobia, specific phobia, OCD, PTSD, etc.; $n=67$ ), only depressive comorbidity (including MDD and dysthymia; $n=16$ ), and both comorbid anxiety and depression $(n=32)$. Two participants had only a comorbid diagnosis that was not an anxiety or depressive disorder (e.g., hypochondriasis) and were excluded from these analyses. Results revealed a significant effect of time $F(1,250)=328.62, p<0.001, \eta_{\mathrm{p}}^{2}=0.57$ and group $F$ $(3,250)=15.03, p<0.001, \eta_{\mathrm{p}}^{2}=0.15$, although, no significant time x group interaction effects were found, $\eta_{\mathrm{p}}^{2}=0.04$ (see Fig. 1). Post hoc tests revealed that individuals with comorbid depression and those with comorbid depression and anxiety had consistently higher PDSS-IE scores. Thus, rates of change across treatment were similar across groups and improvement in PDSS-IE was not a function of comorbidity group status.

\section{Treatment Response and Changes in Comorbidity}

At pretreatment, 117 (45.7\%) individuals had at least one comorbid diagnosis of clinical severity. At posttreatment, only $79(30.9 \%)$ participants were assigned comorbid diagnoses - a reduction that was statistically significant, $\chi^{2}(1, N=256)=49.47, p<0.001, \varphi=0.44$. When examining individual diagnoses, rates of GAD decreased significantly from pretreatment to posttreatment: $25.4 \%$ to $14.1 \%, \chi^{2}$ $(1, N=256)=60.69, p<0.001, \varphi=0.49$. However, this was not true for rates of MDD, $16.4 \%$ to $9.0 \%, \varphi=0.30$; social

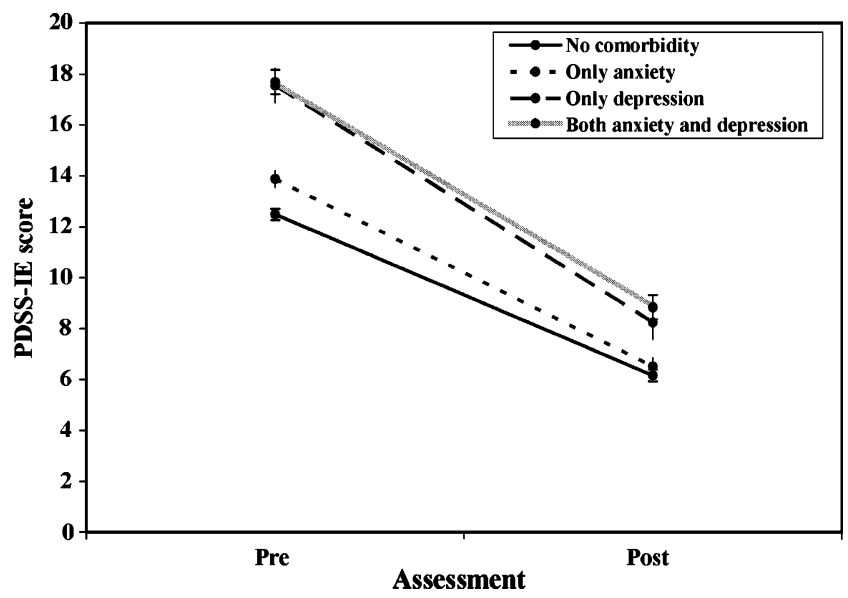

Fig. 1 PDSS-IE scores (with standard errors) across treatment for participants with no comorbidity, only anxiety comorbidity, only depression comorbidity, and both anxiety and depression comorbidity. PDSS-IE panic disorder severity scale - independent evaluator version, Pre pretreatment, Post posttreatment 
phobia, $10.9 \%$ to $6.6 \%, \varphi=0.51$; or specific phobia, $11.7 \%$ to $7.4 \%, \varphi=0.45$.

A 2 (response status: responder or nonresponder) $\times 2$ (time: pre- or posttreatment) repeated measures ANOVA was conducted with each SIGH-A and SIGH-D score as dimensional measures of anxious and depressive symptoms, respectively. These analyses focused on response status as a grouping variable because this variable was the primary indicator of successful treatment, which would allow us to determine the relationship of our measure of treatment outcome to changes in comorbid symptoms. On SIGH-A scores, there was a significant effect of time, $F(1$, $254)=102.76, p<0.001, \eta_{\mathrm{p}}^{2}=0.29$, and a small significant group $\times$ time interaction, $F(1,254)=27.39, p<0.001$, $\eta_{\mathrm{p}}^{2}=0.10$. The group effect was not significant $\left(\eta_{\mathrm{p}}^{2}=\right.$ 0.01). As hypothesized, treatment responders showed greater reductions on SIGH-A scores across treatment as compared to nonresponders. Similarly, on the SIGH-D, there was a small significant effect of time $F(1,254)=$ $51.55, p<0.001, \eta_{\mathrm{p}}^{2}=0.17$, and a small significant interaction $F(1,254)=16.98, p<0.001, \eta_{\mathrm{p}}^{2}=0.06$. Again, responders showed greater reductions across treatment as compared to nonresponders, although the group effect was not significant $\left(\eta_{\mathrm{p}}^{2}=0.05\right)$.

\section{Discussion}

The results from our clinical trial provide support that a structured CBT protocol for panic disorder is similarly efficacious for individuals with and without common comorbid anxiety and unipolar mood disorders. Furthermore, successful implementation of the protocol was associated with reduction in symptoms (although not necessarily diagnoses) of anxiety and depressive disorders not specifically addressed in the treatment protocol. Rates of comorbid GAD declined significantly from pre- to posttreatment, and although rates of MDD, specific phobia, and social phobia declined, these changes were not significant. Not surprisingly, treatment responders showed greater reductions on measures of anxiety and depression across treatment, as compared to nonresponders.

Another important finding was that individuals with comorbid depression (with or without comorbid anxiety) showed similar improvement across treatment on a measure of panic disorder and agoraphobia severity (PDSS-IE), as compared to participants with only comorbid anxiety or no comorbidity at all. These data contradict findings from other studies suggesting that the presence of comorbid depression is associated with worse outcome (Brown et al. 1995; Reif et al. 2000; Steketee et al. 2001), although perhaps the smaller sample sizes in these studies may have been related to the differential findings. Laberge et al.
(1993) found that depression was not associated with worse outcome, despite depressed patients' tendency to minimize gains. Similarly, McLean et al. (1998) found no adverse effects of comorbid depression when treating panic disorder. The results from our investigation provide support that comorbid depression is not associated with worse outcome; however, this rather restricted sample due to exclusionary criteria (e.g., bipolar mood disorders) and non-assessment of Axis II disorders make it difficult to draw strong conclusions. Rates of comorbid MDD also decreased, but this decrease did not reach statistical significance. This may suggest that comorbid depressive disorders are somewhat less "responsive" to CBT for panic disorder, as compared to anxiety disorders such as GAD, or may be an artifact of a somewhat smaller rate of comorbid MDD. Similarly, rates of comorbid social phobia and specific phobia showed improvement that also did not reach statistical significance. Further exploration of the relationship of depression and specific types of anxiety disorders to panic disorder treatment is warranted.

One issue that has proven difficult to reconcile is whether the presence of comorbidity is associated with more severe symptoms of the principal disorder (Berkson 1946). The few studies examining this aspect of comorbidity have yielded conflicting results. Brown et al. (1995) found that individuals with panic disorder and at least one additional diagnosis were rated as having more severe panic symptoms than those without any additional diagnoses. In addition, comorbid individuals evidenced higher ASI and WSAS scores. However, Tsao et al. (1998) found no such difference in their sample of panic disorder patients with and without comorbidity (which may have been due to relatively small sample size), although, in a follow-up investigation, Tsao and colleagues (2002) reported that patients with comorbidity reported significantly higher ASI scores than those without comorbidity. Our study demonstrated that $\mathrm{COM}$ individuals were rated as more severe on all measures of panic disorder, and number of comorbid diagnoses was significantly associated with measures of panic disorder and agoraphobia severity. Given the variety of measures used, and the fact that the data were taken from both patients and independent evaluators, this finding appears robust, at least with respect to the relationship between anxiety and depression comorbidity and panic disorder. Even more encouraging is that, despite having more severe panic disorder symptoms at pretreatment, individuals with comorbid anxiety and/or depression were equally likely to respond to treatment as those without such comorbid diagnoses, at least on categorical measures of treatment response.

There are several possible mechanisms by which CBT for panic disorder may be having an impact on comorbid conditions, including 1) many of the skills taught in CBT for 
panic (e.g., monitoring, cognitive restructuring, and exposure) are useful in coping with other emotional disorders and 2) alleviating panic symptoms as a "cause" for other symptoms of anxiety and depression will necessarily reduce the prevalence and intensity of "secondary" comorbid disorders. A potentially more interesting hypothesis is that CBT may be targeting a broader problem experiencing and regulating emotions (see Barlow et al. 2004; Campbell-Sills and Barlow 2007). CBT, then, may serve to reduce the intensity of all emotional experiences, rather than just panic symptoms.

There are several limitations of the current study worth noting. First, due to the nature of the study, only patients with a principal diagnosis of panic disorder were included. In addition, we did not include an assessment of Axis II disorders, which limits generalizability of the findings to comorbid diagnoses outside of anxiety and mood disorders. Study inclusionary criteria may have also restricted the applicability of the findings to a more diverse patient population. Replication of these findings is needed in more diagnostically and ethnically diverse community and clinical samples. Additionally, there was no control condition in the current study (i.e., all patients received the same treatment), so there can be no guarantee that treatment effects are specifically related to CBT. Finally, the nature of the study design precludes follow-up analyses to explore the relationship of CBT for panic disorder to comorbidity over time.

Nonetheless, findings from the current study add to the knowledge of panic disorder and comorbid anxiety and depression in a number of important ways. These data confirm findings that comorbidity does not necessarily impede treatment for panic disorder, although highlight the possibility of differential relationships between panic disorder and specific comorbid anxiety and depressive disorders. Further research exploring these differential relationships is clearly warranted, particularly with more generous inclusionary criteria, and the relationship of comorbidity and treatment outcome for other disorders.

Acknowledgements This research was supported by National Institute of Mental Health grants R01 MH45963, MH45964, MH45965, and MH45966.

Portions of these data were presented at the 37 th annual convention of the Association for Advancement of Behavior Therapy, Boston, Massachusetts (2003).

Open Access This article is distributed under the terms of the Creative Commons Attribution Noncommercial License which permits any noncommercial use, distribution, and reproduction in any medium, provided the original author(s) and source are credited.

\section{References}

Aaronson, C. J., Shear, M. K., Goetz, R. R., Allen, L. B., Barlow, D. H., White, K. S., et al. (2008). Predictors and time course of response among panic disorder patients treated with cognitivebehavioral therapy. Journal of Clinical Psychiatry, 69, 418-424.

Barlow, D. H., \& Craske, M. G. (2007). Mastery of your anxiety and panic: Workbook (4th ed.). New York: Oxford University Press.

Barlow, D. H., Allen, L. B., \& Choate, M. L. (2004). Towards a unified treatment for emotional disorders. Behavior Therapy, 35, 205-230.

Berkson, J. (1946). Limitations of the application of fourfold table analysis to hospital data. Biometrics Bulletin, 2, 47-53.

Brown, T. A., \& Barlow, D. H. (1992). Comorbidity among anxiety disorders: implications for treatment and DSM-IV. Journal of Consulting and Clinical Psychiatry, 60, 835-844.

Brown, T. A., \& Barlow, D. H. (1995). Long-term outcome in cognitive-behavioral treatment of panic disorder: clinical predictors and alternative strategies for assessment. Journal of Consulting and Clinical Psychiatry, 63, 754-765.

Brown, T. A., Antony, M. M., \& Barlow, D. H. (1995). Diagnostic comorbidity in panic disorder: effect on treatment outcome and course of comorbid diagnoses following treatment. Journal of Consulting and Clinical Psychiatry, 63, 408-418.

Brown, T. A., Campbell, L. A., Lehman, C. L., Grisham, J. R., \& Mancill, R. B. (2001a). Current and lifetime comorbidity of the $D S M-I V$ anxiety and mood disorders in a large clinical sample. Journal of Abnormal Psychology, 110, 585-599.

Brown, T. A., Di Nardo, P. A., Lehman, C. L., \& Campbell, L. A. (2001b). Reliability of DSM-IV anxiety and mood disorders: implications for the classification of emotional disorders. Journal of Abnormal Psychology, 110, 49-58.

Campbell-Sills, L., \& Barlow, D. H. (2007). Incorporating emotion regulation into conceptualizations and treatments of anxiety and mood disorders. In J. J. Gross (Ed.), Handbook of emotion regulation (pp. 542-559). New York: Guilford.

de Ruiter, C., Rijken, H., Garssen, B., van Schaik, A., \& Kraaimaat, F. (1989). Comorbidity among the anxiety disorders. Journal of Anxiety Disorders, 3, 57-68.

Di Nardo, P. A., Brown, T. A., \& Barlow, D. H. (1994). Anxiety disorders interview schedule for DSM-IV. Albany, NY: Graywind.

Guy, W. (1976). ECDEU assessment manual for psychopharmacology, revised (DHEW Publication No. ADM 76-338). Washington, DC: U.S. Government Printing Office.

Hafner, J., \& Marks, I. M. (1976). Exposure in vivo of agoraphobics: contributions of diazepam, group exposure, and anxiety evocation. Psychological Medicine, 6, 71-88.

Hamilton, M. (1959). The assessment of anxiety states by rating. British Journal of Psychiatry, 32, 50-55.

Hamilton, M. (1960). A rating scale for depression. Journal of Neurology, Neurosurgery and Psychiatry, 23, 56-62.

Laberge, B., Gauthier, J. G., Cote, G., Plamondon, J., \& Cormier, H. J. (1993). Cognitive-behavioral therapy of panic disorder with secondary major depression: a preliminary investigation. Journal of Consulting and Clinical Psychiatry, 61, 1028-1037.

Maser, J. D., \& Cloninger, C. R. (eds). (1990). Comorbidity of mood and anxiety disorders. Washington, DC: American Psychiatric.

McLean, P. D., Woody, S., Taylor, S., \& Koch, W. J. (1998). Comorbid panic disorder and major depression: implications for cognitive-behavioral therapy. Journal of Consulting and Clinical Psychiatry, 66, 240-247.

Mundt, J. C., Marks, I. M., Shear, M. K., \& Greist, J. H. (2002). The work and social adjustment scale: a simple measure of impairment in functioning. British Journal of Psychiatry, 180, 461-464.

Peterson, R. A., \& Reiss, S. (1987). Anxiety sensitivity index manual. Worthington, OH: IDS.

Reif, W., Trenkamp, S., Auer, C., \& Fichter, M. M. (2000). Cognitive behavioral therapy in panic disorder and comorbid major depression. Psychotherapy and Psychosomatics, 69, 70-78. 
Sanderson, W. C., Di Nardo, P. A., Rapee, R. M., \& Barlow, D. H. (1990). Syndrome comorbidity in patients diagnosed with a DSM-III-R anxiety disorder. Journal of Abnormal Psychology, 99, 308-312.

Shear, M. K., Brown, T. A., Sholomskas, D. E., Barlow, D. H., Gorman, J. M., Woods, S. W., et al. (1992). Panic disorder severity scale (PDSS). Pittsburgh, PA: Department of Psychiatry, University of Pittsburgh School of Medicine.

Shear, M. K., Brown, T. A., Barlow, D. H., Money, R., Sholomskas, D. E., Woods, S. W., et al. (1997). Multicenter collaborative panic disorder severity scale. American Journal of Psychiatry, 154, 1571-1575.

Shear, M. K., Vander Bilt, J., Rucci, P., Endicott, J., Lydiard, B., Otto, M. W., et al. (2001). Reliability and validity of a structured interview guide for the Hamilton anxiety rating scale (SIGH-A). Depression and Anxiety, 13, 166-178.
Steketee, G., Chambless, D. L., \& Tran, G. Q. (2001). Effects of Axis I and II comorbidity on behavior therapy outcome for obsessivecompulsive disorder and agoraphobia. Comprehensive Psychiatry, $42,76-86$.

Tsao, J. C. I., Lewin, M. R., \& Craske, M. G. (1998). The effects of cognitive-behavior therapy for panic disorder on comorbid conditions. Journal of Anxiety Disorders, 12, 357-371.

Tsao, J. C. I., Mystkowski, J. L., Zucker, B. G., \& Craske, M. G. (2002). Effects of cognitive-behavior therapy for panic disorder on comorbid conditions: replication and extension. Behavior Therapy, 33, 493-509.

Williams, J. B. (1988). A structured interview guide for the Hamilton depression rating scale. Archives of General Psychiatry, 45, 742747. 